\title{
Hand knob stroke from cancer-associated thromboembolism
}

\author{
Yoshitaka Tomoda MD PhD, Masahito Tanaka MD PhD, Kazutoyo Tanaka MD
}

Cite as: CMAJ 2019 October 21;191:E1164. doi: 10.1503/cmaj.190673

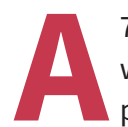

72-year-old man undergoing chemotherapy with 4 cycles of gemcitabine and nabpaclitaxel for pancreatic ductal adenocarcinoma, diagnosed 5 months previously, presented to the emergency department with sudden difficulty moving his left hand. Neurological examination showed paresis of extension of the left wrist. Weakness of all fingers of the left hand was present, including a teardrop sign from paresis of the finger flexors (Figure 1A). Sensory examination results were normal. Diffusion-weighted brain magnetic resonance imaging (MRI) showed an acute infarction in the superior part of the patient's right precentral gyrus, called the hand knob area (Figures 1B, 1C). No substantial internal carotid artery stenosis was visualized on MRI angiography. Contrast-enhanced full-body computed tomography, obtained for suspected thrombosis, showed the pancreatic tumour, unchanged in size, and pulmonary embolism in the right pulmonary artery (Figures 1D, 1E). After careful exclusion of cardioembolism through cardiac monitoring and transthoracic echocardiogram, we diagnosed stroke in the hand knob area, from cancer-associated embolism. The patient's symptoms gradually improved after treatment with lowmolecular-weight heparin (LMWH).

Infarction in the hand knob area, the site of hand motor function, can cause isolated upper limb paresis and may be misdiagnosed as peripheral nerve damage. This is an uncommon presentation of acute stroke, with an incidence rate of $<1 \%$ among all ischemic strokes. ${ }^{1}$ This manifestation is typically associated with atheroembolism or cardioembolism.

Patients with malignant disease are in a hypercoagulable state and at risk for the development of thromboembolism that may be arterial or venous in origin. Pathogenesis of the hypercoagulable state of malignancy has not been fully elucidated, but involves the interplay of multiple mechanisms, including those associated with carcinoma mucins, tissue factor and cancer procoagulant. ${ }^{2}$ Standard treatment includes early initiation of LMWH, with direct oral anticoagulants being a preferable alternative. Although hand knob infarction has a good functional outcome, cancer-associated thromboembolism has a higher incidence of recurrence and is the second leading cause of death in patients with cancer. ${ }^{3}$

\section{References}

1. Celebisoy M, Ozdemirkiran T, Tokucoglu F, et al. Isolated hand palsy due to cortical infarction: localization of the motor hand area. Neurologist 2007;13:376-9.

2. Abdol Razak NB, Jones G, Bhandari M, et al. Cancer-associated thrombosis: an overview of mechanisms, risk factors, and treatment. Cancers (Basel) 2018;10:pii: E380.

3. Khorana AA. Venous thromboembolism and prognosis in cancer. Thromb Res 2010;125:490-3.

\section{Competing interests: None declared.}

This article has been peer reviewed.

The authors have obtained patient consent.

Affiliations: Departments of General Medicine (Tomoda, K. Tanaka) and Neurology (M. Tanaka), Saiseikai Fukuoka General Hospital, Fukuoka, Japan.

Correspondence to: Yoshitaka Tomoda, yoshisoph@gmail.com 